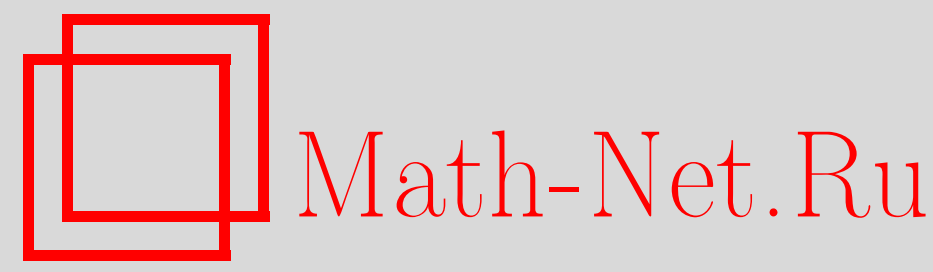

В. М. Мануйлов, Диагонализация операторов над непрерывными полями $C^{*}$ алгебр, Матем. сб., 1997, том 188, номер 6, 99-118

DOI: https://doi.org/10.4213/sm229

Использование Общероссийского математического портала Math-Net.Ru подразумевает, что вы прочитали и согласны с пользовательским соглашением

http://www . mathnet.ru/rus/agreement

Параметры загрузки:

IP: 54.224 .187 .69

26 апреля 2023 г., $14: 39: 58$ 
УДК 517.98

\author{
В. М. Мануйлов
}

\title{
Диагонализация операторов над непрерывными полями $C^{*}$-алгебр
}

\begin{abstract}
В работе доказывается некомму тативный аналог классической теоремы Гильберта-Шмидта о приведении самосопряженного компактного оператора в гильбертовом пространстве к диагональному виду. Именно, для некоторого класса $C^{*}$-алгебр доказано, что самосопряженный компактньпй оператор в гильбертовом модуле $H_{A}$ над $C^{*}$-алгеброй $A$ приводится в некотором большем модуле над большей $W^{*}$-алгеброй к диагональному виду с элементами алгебры $A$ на диагонали.

Библиограффия: 25 названий.
\end{abstract}

\section{Введение}

Пусть $X$ есть компактное хаусдорфово пространство, $\{A(x), x \in X\}$ - семейство $C^{*}$-алгебр с единицей, снабженных точными конечными следами $\tau_{x}, \tau_{x}\left(1_{x}\right)=1$. Через $\prod_{x \in X} A(x)$ обозначим множество функций $a=a(x)$, определенных на $X$, и таких, что $a(x) \in A(x)$ для всех $x \in X$.

ОПРЕДЕЛЕНИЕ 0.1. Пусть $\mathscr{A} \subset \prod_{x \in X} A(x)$ есть подмножество со следующими свойствами:

i) $\mathscr{A}$ есть $*$-подалгебра в $\prod_{x \in X} A(x)$;

ii) для любого $x \in X$ множество $\{a(x), a \in \mathscr{A}\}$ плотно в алгебре $A(x)$;

iii) для каждого $a \in \mathscr{A}$ функция $x \mapsto\|a(x)\|$ непрерывна;

iv) пусть $a \in \prod_{x \in X} A(x)$; если для каждого $x \in X$ и для всех $\varepsilon>0$ существует такой элемент $a^{\prime} \in \mathscr{A}$, что $\left\|a(x)-a^{\prime}(x)\right\|<\varepsilon$ в некоторой окрестности точки $x$, то $a \in \mathscr{A}$;

v) для каждого $a \in \mathscr{A}$ функция $x \mapsto \tau_{x}(a(x))$ непрерьвна.

Тогда тройка $(A(x), X, \mathscr{A})$ называется непрерывным полем $C^{*}$-алгебр со следом.

Отметим, что первые четыре свойства этого определения составляют стандартное определение непрерывного поля $C^{*}$-алгебр [1]. Известно, что $\mathscr{A}$ является $C^{*}$-алгеброй. Для краткости через $\|a(x)\|$ мы будем обозначать норму элемента $a=a(x) \in \mathscr{A}$, т.е. $\sup _{x}\|a(x)\|$. Если $V \subset X$ - замкнутое подмножество, то через $\left.\mathscr{A}\right|_{V}$ мы будем обозначать ограничение непрерьвного поля $C^{*}$-алгебр на $V$.

Очевидно, что $\tau: a \mapsto \tau_{x}(a(x))-$ след на $\mathscr{A}$, принимающий значения в $C(X) \subset \mathscr{A}$.

Работа вьполнена при частичной поддержке Российского фонда фундаментальных исследований (грант № 96-01-00276) и КЦФЕ. 
Этот след задает скалярное произведение на $A(x)$

$$
\left(b_{1}, b_{2}\right)_{\tau_{x}}=\tau_{x}\left(b_{1}^{*} b_{2}\right), \quad\|b\|_{\tau_{x}}^{2}=(b, b)_{\tau_{x}},
$$

и $C(X)$-значное внутреннее произведение на $\mathscr{A}$

$$
\left(b_{1}(x), b_{2}(x)\right)_{\tau}=\tau_{x}\left(b_{1}^{*}(x) b_{2}(x)\right) \in C(X)
$$

с нормой

$$
\|b(x)\|_{\tau}=\sup _{x}(b(x), b(x))_{\tau}^{1 / 2}=\sup _{x}\|b(x)\|_{\tau_{x}} .
$$

Пусть $H(x)=L^{2}(A(x))$ есть пополнение $A(x)$ по норме $\|\cdot\|_{\tau_{x}}$. Тогда алгебра $A(x)$ является точно представленной в гильбертовом пространстве $H(x)$, и мы можем перейти к соответствуюшей $W^{*}$-алгебре $B(x)=L^{\infty}(A(x))$, т.е. к слабому замыканию $A(x)$ (обозначение $L^{\infty}$ выбрано по аналогии с коммутативным случаем).

Пусть теперь $d x$ есть $\sigma$-конечная борелевская мера на $X$. Отметим, что функция (0.1) непрерывна, поэтому можно положить

$$
\left(b_{1}, b_{2}\right)_{\tau}=\int_{X}\left(b_{1}(x), b_{2}(x)\right)_{\tau_{x}} d x, \quad\|b\|_{\tau}^{2}=(b, b)_{\tau} .
$$

Обозначим пополнение алгебры $\mathscr{A}$ по этой норме через $H=L^{2}(\mathscr{A})$. Алгебра $\mathscr{A}$ точно представлена в гильбертовом пространстве $H$, и соответствующую $W^{*}$-алгебру мы обозначим через $\mathbf{A}=L^{\infty}(\mathscr{A}) \supset \mathscr{A}$. Очевидно, что

$$
\mathbf{A}=\int_{X}^{\oplus} B(x) d x
$$

Из точности и конечности следа, определенного семейством следов $\tau_{x}$ на $\mathbf{A}$ и принимающего значения в $L^{\infty}(X)$, следует, что алгебра $\mathbf{A}$ является конечной $W^{*}$-алгеброй с конечньп точным следом $\bar{\tau}=\int_{X} \tau_{x} d x$.

Далее мы будем иметь дело только со случаем, когда $X$ есть отрезок или окружность. Тогда если $X$ разделен точками $\left\{x_{k}\right\}$ на отрезки $D_{k}=\left[x_{k}, x_{k+1}\right]$ и если $\left.a_{k}(x) \in \mathscr{A}\right|_{D_{k}}$ есть непрерывное поле для каждого $k$, то мы будем назьвать множество $a(x)=\left\{a_{k}(x)\right\}$ кусочно-непрерывным полем на $X$. Такие кусочно-непрерывные поля, очевидно, принадлежат $W^{*}$-алгебре А. Расстояние от такого поля до $C^{*}$-алгебры $\mathscr{A}$ задается формулой

$$
\operatorname{dist}(a(x), \mathscr{A})=\sup _{k}\left\|a_{k}\left(x_{k}\right)-a_{k+1}\left(x_{k}\right)\right\| .
$$

Для удобства обозначений мы будем писать $K_{1}(x) \sim K_{2}(x)$ для двух кусочно-непрерывных полей $K_{1}(x)$ и $K_{2}(x)$, если существует кусочно-непрерывное унитарное поле $u(x)$ такое, что $u^{*}(x) K_{1}(x) u(x)=K_{2}(x)$.

Статья состоит из четырех параграфов. В первом параграфе мы обсуждаем понятие диагонализируемости для операторов, действующих в гильбертовых $C^{*}$-модулях. 
В $\S 2$ мы обсуждаем одну проблему, связанную с почти коммутирующими операторами, и доказываем для некоторого класса $C^{*}$-алгебр (включая матричные), что если $h \in A$ есть самосопряженный элемент, $u \in A$ - унитарный, и норма их коммутатора $[u, h]$ достаточно мала, то $u$ можно соединить с единицей непрерывным путем $u(t)$ так, чтобы норма коммутатора $[u(t), h]$ была бы мала вдоль этого пути. Этот факт используется для доказательства основной теоремы, но он представляет также независимый интерес в связи с топологическими приложениями [2]. Оценка, которую мы даем для нормы коммутатора, не является наилучшей, но она достаточна для наших целей.

Параграф 3 содержит доказательство диагонализируемости самосопряженных компактных операторов в гильбертовых $C^{*}$-модулях над некоторыми непрерывными полями $C^{*}$-алгебр нулевого вещественного ранга со следом над одномерньпи многообразиями. К сожалению, наш метод не может быть применен в случае произвольной размерности базы, хотя мы предполагаем, что наш результат верен в большей обшности. Условия, которым должны удовлетворять $C^{*}$-алгебры - слои, также далеки от необходимых и могут быть ослаблены в различных направлениях.

В последнем параграфе мы приводим пример $C^{*}$-алгебры, не допускающей непрерывной диагонализации самосопряженных операторов.

Автор выражает благодарность А. С. Мищенко, Е. В. Троицкому и М. Франку за полезные обсуждения.

\section{§1. Диагонализация операторов в гильбертовых $C^{*}$-модулях}

Пусть $\mathscr{A}$ есть $C^{*}$-алгебра с единицей, $H_{\mathscr{A}}$ - правый гильбертов $\mathscr{A}$-модуль последовательностей $a=\left(a_{k}\right), a_{k} \in \mathscr{A}, k \in \mathbb{N}$, таких, что ряд $\sum a_{k}^{*} a_{k}$ сходится в $\mathscr{A}$ по норме; в $H_{\mathscr{A}}$ введем стандартньй базис $\left\{e_{k}\right\}$. Внутреннее $\mathscr{A}$-значное произведение на модуле $H_{\mathscr{A}}$ задается формулой $\langle x, y\rangle=\sum x_{k}^{*} y_{k}, x, y \in \mathscr{A}$. Обозначим через $L_{n}(\mathscr{A}) \subset H_{\mathscr{A}}$ подмодуль, порожденный первыми $n$ элементами $e_{1}, \ldots, e_{n}$ базиса. Теория гильбертовых $C^{*}$-модулей и операторов в них подробно изложена в статьях [3]-[8] и в книге [9].

Через $H_{\mathscr{A}}^{*}=\operatorname{Hom}_{\mathscr{A}}\left(H_{\mathscr{A}} ; \mathscr{A}\right)$ обозначим $\mathscr{A}$-модуль, дуальный к $H_{\mathscr{A}}$, т.е. состоящий из всех ограниченных $\mathscr{A}$-линейных $\mathscr{A}$-значных функционалов на $H_{\mathscr{A}}$. Напомним, что модуль $L_{n}(\mathscr{A})$ является автодуальнылм, т.е. $L_{n}^{*}(\mathscr{A})=L_{n}(\mathscr{A})$. Ограниченный оператор $K: H_{\mathscr{A}} \rightarrow H_{\mathscr{A}}$ называется компактным [6] [7], если он лежит в замькании (по норме) линейной оболочки операторов вида

$$
\theta_{x, y}, \quad \theta_{x, y}(z)=x\langle y, z\rangle, \quad x, y, z \in H_{\mathscr{A}} .
$$

Мы называем компактный оператор $K$ строго положительным, если $\langle K x, x\rangle$ является положительным элементом в $\mathscr{A}$, и если его спектральный проектор, отвечающий нулевой точке спектра $K$, является нулевым. Мы используем известньй факт [4], что в случае, когда $\mathscr{A}$ есть $W^{*}$-алгебра, внутреннее произведение естественным образом продолжается на дуальный модуль $H_{\mathscr{A}}^{*}$. Пусть $M_{\mathscr{A}}$ в дальнейшем обозначает либо $L_{n}(\mathscr{A})$, либо $H_{\mathscr{A}}$.

ОПреДЕЛЕНИЕ 1.1. Пусть $\mathbf{A}$ есть $W^{*}$-алгебра. Назовем самосопряженный оператор $K$ в А-модуле $M_{\mathbf{A}}$ диагонализируемым, если существует множество $\left\{x_{i}\right\}$ элементов из $M_{\mathbf{A}}^{*}$ и множество элементов $\lambda_{i} \in \mathbf{A}$ таких, что

i) множество $\left\{x_{i}\right\}$ ортонормированно, т.е. $\left\langle x_{i}, x_{j}\right\rangle=\delta_{i j}$; 
ii) модуль $M_{\mathbf{A}}^{*}$ совпадает с $\mathbf{A}$-модулем $\mathscr{M}^{*}$, дуальным к модулю $\mathscr{M}$, порожденному множеством $\left\{x_{i}\right\}$;

iii) $K x_{i}=x_{i} \lambda_{i}$;

iv) для любых унитарных элементов $u_{i}, u_{i+1} \in \mathbf{A}$ выполнено операторное неравенство

$$
u_{i}^{*} \lambda_{i} u_{i} \geqslant u_{i+1}^{*} \lambda_{i+1} u_{i+1}
$$

Элементы $x_{i}$ мы называем “собственными векторами", а операторы $\lambda_{i}-$ "собственными значениями" оператора $K$. Отметим, что "собственные векторы" и "собственные значения" определены неоднозначно. Условие iv) означает, что наше определение диагонализируемости включает в себя естественное упорядочение "собственных значений".

Задача диагонализации операторов в гильбертовых модулях впервые была поставлена Р. В. Кэдисоном в [10], где доказано, что самосопряженный оператор в модуле $L_{n}(\mathbf{A})$ над $W^{*}$-алгеброй $\mathbf{A}$ диагонализируем. Далее эта задача обсуждалась в различных ситуациях в [11]-[14] и т. д. В работах [15], [16] нами была доказана следуюшая

Теорема 1.2. Если $\mathbf{A}$ есть конечная $W^{*}$-алгебра с конечным нормальным точным следом, то компактный строго положстельный оператор $К$ в модуле $H_{\mathbf{A}}$ может бъть диагонализирован в $H_{\mathbf{A}}^{*}$, и его “собственные значения" определень единственным образом с точностью до унитарной әквивалентности.

В работе [17] было также доказано, что "собственные значения" непрерьвно зависят от компактных операторов, точнее

Теорема 1.3. Если $K_{k}: H_{\mathbf{A}} \rightarrow H_{\mathbf{A}}, k=1,2$, суть компактные строго положительные операторы и если $\left\|K_{1}-K_{2}\right\|<\varepsilon$, то

i) существует унитарный оператор $U$, действующий на модуле $H_{\mathbf{A}}^{*}$, отображающий “собственные векторы" $K_{2}$ в "собственные векто$p u$ " $K_{1},\left\|U^{*} K_{1} U-K_{2}\right\|<\varepsilon$

ii) “собственные значения" $\left\{\lambda_{i}^{(k)}\right\}$ операторов $K_{k}, k=1,2$, можсно вибрать так, что $\left\|\lambda_{i}^{(1)}-\lambda_{i}^{(2)}\right\|<\varepsilon$.

Известно, что в коммутативном случае, т.е. когда $\mathscr{A}=C(X)$ есть $C^{*}$-алгебра непрерывных функций на компакте, компактные самосопряженные операторы не могут быть диагонализированы в модуле $H_{\mathscr{A}}$. Однако это становится возможным в большем модуле над большей $W^{*}$-алгеброй $L^{\infty}(X)=\mathbf{A} \supset \mathscr{A}$, которая получается из $\mathscr{A}$ ее пополнением (на ограниченных множествах) по слабой топологии в естественном представлении $\mathscr{A}$ в гильбертовом пространстве $H=L^{2}(X)$ с интегральной нормой, задаваемой точным конечньм следом (мерой) на $\mathscr{A}$. В отличие от "собственных векторов", “собственные значения" являются непрерьвньми, т.е. лежат в исходной $C^{*}$-алгебре $\mathscr{A}$. Это приводит нас к следуюшему определению.

Пусть $\mathscr{A}$ есть $C^{*}$-алгебра с конечным точным следом $\tau$. Пусть $(a, b)_{\tau}=\tau\left(a^{*} b\right)$, $a, b \in \mathscr{A}$, есть невырожденное скалярное произведение на $\mathscr{A}$, а $\|\cdot\|_{\tau}=(\cdot, \cdot)_{\tau}^{1 / 2}$ - норма, задаваемая следом $\tau$ на $\mathscr{A}$. Пополняя $\mathscr{A}$ по этой норме, мы получаем 
гильбертово пространство $L^{2}(\mathscr{A})$ и точное представление $\mathscr{A}$ в этом пространстве. Пусть $\mathbf{A}=L^{\infty}(\mathscr{A})$ есть соответствующая конечная $W^{*}$-алгебра со следом, содержащая $\mathscr{A}$ как слабо плотную подалгебру. Пусть $K$ есть компактньй строго положительный оператор в $H_{\mathscr{A}}$. Мы можем естественным образом продолжить $K$ на больший модуль $H_{\mathbf{A}}^{*}$, в котором, как легко проверить, он по-прежнему будет компактным и строго положительным, и в котором он может быть диагонализирован по теореме 1.2.

ОПРеДЕЛЕНИЕ 1.4. Назовем $C^{*}$-алгебру $\mathscr{A}$ допускающей слабую диагонализаиию, если для любого компактного строго положительного оператора $K$ в $H_{\mathscr{A}}$ диагональные элементы (после диагонализации в $H_{\mathbf{A}}^{*}$ ) могут быть выбраны в $\mathscr{A}$ (вместо A).

ПроБЛЕмА. Описать класс $C^{*}$-алгебр с конечным точным следом, допускающих слабую диагонализачию.

Отметим, что если $C^{*}$-алгебра допускает слабую диагонализацию, то каждьй самосопряженный оператор конечного ранга $K \in M_{n} \otimes \mathscr{A}$ также может быть диагонализирован над $W^{*}$-алгеброй $\mathbf{A}$ c “собственными значениями”, лежащими в $\mathscr{A}$.

Напомним, что вешественный ранг нуль $(R R(\mathscr{A})=0)$ означает, что каждый самосопряженный оператор в $\mathscr{A}$ допускает аппроксимацию операторами с конечным спектром, т.е. имеющими вид $\sum \alpha_{i} p_{i}$, где $p_{i} \in \mathscr{A}$ суть самосопряженные взаимно ортогональные проекторы, а $\alpha_{i} \in \mathbb{R}[18]$. Согласно [18] из условия $R R(\mathscr{A})=0$ следует, что $R R\left(\operatorname{End}_{\mathscr{A}}\left(L_{n}(\mathscr{A})\right)\right)=0$. В работе [17] было показано, что наряду с коммутативньми $C^{*}$-алгебрами класс $C^{*}$-алгебр, допускающих слабую диагонализацию, включает также $C^{*}$-алгебры нулевого вещественного ранга со следом, обладающие следующим свойством:

(*) для каждых двух проекторов $p, q \in \mathscr{A}$ в $\mathscr{A}$ сушествуют эквивалентные друг другу (в $\mathscr{A})$ проекторы $r_{p} \sim r_{q}, r_{p} \leqslant p, r_{q} \leqslant q$ такие, что $T\left(r_{p}\right)=T\left(r_{q}\right)=$ $\min \{T(p)(z), T(q)(z)\}, z \in Z$, где $Z$ есть пространство максимальных идеалов центра $\mathscr{Z} W^{*}$-алгебры $L^{\infty}(A)$, а $T$ - стандартный $\mathscr{Z}$-значный след на $L^{\infty}(A)$.

Это свойство означает, что естественное отображение $K_{0}(\mathscr{A}) \rightarrow K_{0}\left(L^{\infty}(\mathscr{A})\right)$ является вложением, и $K_{0}(\mathscr{A})$ является подрешеткой в $K_{0}\left(L^{\infty}(\mathscr{A})\right)$. Кроме конечных факторов указанным свойством обладают $C^{*}$-алгебры иррационального врашения [19] и алгебры Бунса - Дедденса [20].

\section{§2. О почти коммутирующих операторах}

Следующий результат о почти коммутирующих операторах в некоторых $C^{*}$-алгебрах (в том числе матричных) мы используем для диагонализации непрерывных полей операторов. Напомним, что $\operatorname{tsr}(A)=1$ означает, что обратимые элементы плотны в $A$.

ПРЕДЛОЖЕНИЕ 2.1. Пусть $A$ есть $C^{*}$-алгебра со свойствами:

i) $R R(A)=0 u \operatorname{tsr}(A)=1$;

ii) для любого проектора $p \in A$ унитарная группа $C^{*}$-алгебры рАр связна. 
Пусть $h \in A$ есть самосопряженный, $u \in A-$ унитарный әлемент такие, что

$$
\left\|u^{*} h u-h\right\|<\delta .
$$

Тогда существуют константь $C$ и $\delta_{0}>0$, зависящие только от $\|h\|, u$ непрерывный унитарный путь $и(t)$, соединяющий и с единицей, такой, что для $\delta<\delta_{0}$ при всех значениях параметра $t$ выполнено неравенство

$$
\left\|u^{*}(t) h u(t)-h\right\|<C \sqrt[4]{\delta}
$$

ДокАЗАТЕЛьство. Поскольку $R R(A)=0$, без ограничения обшности можем предположить, что оператор $h$ является линейной комбинацией взаимно ортогональных проекторов, $h=\sum_{i=1}^{n} \lambda_{i} p_{i} \in A$ с вещественными собственными значениями $\lambda_{i}$. Мы можем также предположить, что эти собственные значения упорядочены, т.е. $\lambda_{1}>\cdots>\lambda_{n}$. Разобьем отрезок $\left[\lambda_{n}, \lambda_{1}\right]$ на менњшие отрезки длины $\sqrt[4]{\delta}$ и пронумеруем только те отрезки, которые содержат хотя бы одно собственное значение $\lambda_{i}$. Обозначим эти отрезки через $\Delta_{k}$. Число $m$ таких отрезков не превосходит $\left(\lambda_{1}-\lambda_{n}\right) / \sqrt[4]{\delta}+1$. При этом выполнены следующие свойства:

i) если $\lambda_{i}, \lambda_{j} \in \Delta_{k}$, то $\left|\lambda_{i}-\lambda_{j}\right|<\sqrt[4]{\delta}$;

ii) если $\lambda_{i} \in \Delta_{k-1}$ и $\lambda_{j} \in \Delta_{k+1}$, то $\left|\lambda_{i}-\lambda_{j}\right| \geqslant \sqrt[4]{\delta}$.

Обозначим через $\mu_{k}$ середины отрезков $\Delta_{k}$. Тогда если $\mu_{k+1}-\mu_{k}>\sqrt[4]{\delta}$, то спектр $h$ имеет лакуну длины не менее, чем $\sqrt[4]{\delta}$.

Обозначим спектральные проекторы оператора $h$, отвечаюшие отрезкам $\Delta_{k}$, через $q_{k}, q_{1}+\cdots+q_{m}=1$ (если точка спектра $\lambda_{j}$ совпадает с граничной точкой двух соседних отрезков $\Delta_{k}$ и $\Delta_{k+1}$, отнесем ее спектральный проектор произвольно либо к $q_{k}$, либо к $q_{k+1}$ ). Тогда алгебра $A$ как $A$-модуль может быть разложена в прямую сумму, отвечающую этим проекторам, $A=\bigoplus_{k=1}^{m} q_{k} A$. Будем записывать элементы алгебры $A$ в виде матриц по отношению к этому разложению: $h=\operatorname{diag}\left(\left\{h_{i}\right\}\right), h_{i}=q_{i} h q_{i}$, и $u=\left(u_{i j}\right), u_{i j}=q_{i} u q_{j}$.

Заметим, что если $m=1$, т.е. если все собственные значения $h$ отличаются друг от друга не более, чем на $\sqrt[4]{\delta}$, то мы можем взять любой унитарньй путь $u(t)$, соединяющий $u$ с единицей. Поскольку при $m=1$ существует число $\mu$ такое, что $\|h-\mu\|<\sqrt[4]{\delta} / 2$, и следовательно,

$$
\left\|v^{*} h v-h\right\| \leqslant\left\|v^{*} h v-v^{*} \mu v\right\|+\|\mu-h\|<2 \frac{\sqrt[4]{\delta}}{2}=\sqrt[4]{\delta}
$$

для любого унитарного $v$, то далее мы можем предполагать, что $m>1$.

Рассмотрим еше одно разбиение спектра оператора $h$ на более мелкие (чем $\Delta_{k}$ ) отрезки $\bar{\Delta}_{s}$ длины $\delta$. Как и раньше, мы принимаем в рассмотрение только такие отрезки $\bar{\Delta}_{s}$, которые содержат хотя бы одну точку спектра оператора $h$. Без ограничения общности можно считать, что каждый отрезок $\bar{\Delta}_{s}$ лежит в одном из отрезков $\Delta_{k}$. Пусть $\bar{\lambda}_{s}$ суть середины отрезков $\bar{\Delta}_{s}$ и пусть $\bar{p}_{s}-$ спектральные проекторы $h$, отвечающие отрезкам $\bar{\Delta}_{s}$. Положим

$$
\bar{h}=\sum_{s} \bar{\lambda}_{s} \bar{p}_{s}
$$


Очевидно, $\|h-\bar{h}\|<\delta / 2$, поэтому из (2.1) следует, что

$$
\left\|u^{*} \bar{h} u-\bar{u}\right\| \leqslant\left\|u^{*} \bar{h} u-u^{*} h u\right\|+\left\|u^{*} h u-h\right\|+\|h-\bar{h}\|<2 \delta .
$$

Пусть $A=\bigoplus_{s} \bar{p}_{s} A$ есть разложение $A$ в прямую сумму, отвечающее спектральным проекторам $h$. Оно является подразложением $\bigoplus_{k=1}^{m} q_{k} A$, и матрица $u$ может быть записана в виде $u=\left(v_{k l}\right), v_{k l}=\bar{p}_{k} u \bar{p}_{l}$, а матричные элементы $u_{i j}$ могут рассматриваться как блоки элементов $v_{k l}$. Пусть $N$ есть число столбцов матрицы $\left(v_{k l}\right)$. Тогда

$$
N<\frac{2\|h\|}{\delta}+1
$$

Перейдем теперь к построению гомотопии $u \sim 1$. Сначала мы определим путь $u(t)$, не являющийся унитарным, но проходящий в окрестности унитарной группы $U(A) C^{*}$-алгебры $A$.

Первый шаг гомотопии. Пусть $a=\left(a_{i j}\right)$ есть матрища, отвечающая прямой сумме $\bigoplus_{k=1}^{m} q_{k} A$. Обозначим через $d_{0}(a)$ ее главную диагональ $\operatorname{diag}\left(\left\{a_{i i}\right\}\right)$, а через $d_{k}(a)$ - диагональ, лежашую на $k$ линий над (или под, если $k$ отрицательно) главной диагональю. Покажем, что матрица $u=\left(u_{i j}\right)$ "почти" трехдиагональна.

Пусть $k_{i}$ и $k_{j}$ суть номера отрезков $\Delta_{k}$, содержаших собственные значения $\bar{\lambda}_{i}$ и $\bar{\lambda}_{j}$ оператора $\bar{h} ; \bar{\lambda}_{i} \in \Delta_{k_{i}}, \bar{\lambda}_{j} \in \Delta_{k_{j}}$. Определим трех диагональную матрицу $d(a)$ следуюшим образом:

i) если $k_{j} \geqslant k_{i}+2$ или $k_{j} \leqslant k_{i}-2$, то положим $(d(a))_{i j}=0$;

ii) если $k_{j}=k_{i} \pm 1$ и $\left|\mu_{k_{j}}-\mu_{k_{i}}\right|>\sqrt[4]{\delta}$, то положим также $(d(a))_{i j}=0$;

iii) в остальных случаях положим $(d(a))_{i j}=a_{i j}$.

Лемма 2.2. Пусть $a=\left(a_{i j}\right), a_{i j} \in A$, есть $(N \times N)$-матрица такая, что для каждого і выполнена оценка $\left\|\sum_{j} a_{i j} a_{i j}^{*}\right\|<\varepsilon^{2}$. Тогда $\|a\|<\varepsilon \sqrt{N}$.

Доказательство. Пусть $\xi=\left(\xi_{k}\right), k=1, \ldots, N, \xi_{i} \in A$. Используя обобщенное неравенство Коши-Шварца [9], получаем, что

$$
\begin{aligned}
\|a \xi\|^{2} & =\left\|\sum_{i j k} \xi_{j}^{*} a_{i j}^{*} a_{i k} \xi_{k}\right\| \leqslant \sum_{i}\left\|\sum_{k j} \xi_{j}^{*} a_{i j}^{*} a_{i k} \xi_{k}\right\| \\
& \leqslant \sum_{i}\left\|\sum_{k} a_{i k} a_{i k}^{*}\right\| \cdot\left\|\sum_{k} \xi_{k}^{*} \xi_{k}\right\|<N \varepsilon^{2}\|\xi\|^{2} .
\end{aligned}
$$

Следовательно, $\|a \xi\|<\sqrt{N} \varepsilon\|\xi\|$.

ЛЕмма 2.3. Из неравенства (2.1) следует, что для достаточно мальх $\delta$ выполнено неравенство

$$
\|u-d(u)\|<4\|h\|^{1 / 2} \sqrt[4]{\delta}
$$


ДокАЗАТЕЛЬСтво. Рассмотрим матрицу $(\bar{h} u-u \bar{h})(\bar{h} u-u \bar{h})^{*}$. Из оценки $(2.2)$ следует, что норма этой матрицы меньше, чем $4 \delta^{2}$, поэтому норма любого матричного элемента этой матрицы также меншше $4 \delta^{2}$. Поскольку $\bar{\lambda}_{j}$ коммутирует с $v_{k j}$, то для каждого $i$

$$
\left\|\sum_{j=1}^{N}\left(\bar{\lambda}_{i}-\bar{\lambda}_{j}\right)^{2} v_{i j} v_{i j}^{*}\right\|<4 \delta^{2} .
$$

Пусть $\bar{\lambda}_{i} \in \Delta_{k_{i}}, \bar{\lambda}_{j} \in \Delta_{k_{j}}$. Так как все слагаемые в (2.4) положительны, опустив часть из них, мы не увеличим нормы суммы, поэтому

$$
\left\|\sum_{j}^{\prime}\left(\bar{\lambda}_{i}-\bar{\lambda}_{j}\right)^{2} v_{i j} v_{i j}^{*}\right\|<4 \delta^{2}
$$

где сумма $\sum^{\prime}$ берется только по тем $j$, для которых либо $\left|k_{j}-k_{i}\right| \geqslant 2$, либо $\left|k_{j}-k_{i}\right|=1$ и $\left|\mu_{k_{j}}-\mu_{k_{i}}\right|>\sqrt[4]{\delta}$, т.е. мы отбрасываем те $v_{i j}$, для которых $(u-d(u))_{i j}=0$. Поэтому в сумме $\sum^{\prime}$ остаются лишш те слагаемые, для которых $\left|\bar{\lambda}_{k}-\bar{\lambda}_{j}\right| \geqslant \sqrt[4]{\delta}$, следовательно,

$$
4 \delta^{2}>\left\|\sum_{j}^{\prime}\left(\bar{\lambda}_{i}-\bar{\lambda}_{j}\right)^{2} v_{i j} v_{i j}^{*}\right\| \geqslant \sqrt{\delta}\left\|\sum_{i}^{\prime} v_{i j} v_{i j}^{*}\right\|
$$

и, значит,

$$
\left\|\sum_{j}^{\prime} v_{i j} v_{i j}^{*}\right\|<4 \delta^{3 / 2} .
$$

Суммы $\sum^{\prime}$ отвечают блокам, лежащим в матрице $u-d(u)$. Из леммы $2.2 \mathrm{c}$ учетом (2.3) и $(2.5)$ (для $\left.\varepsilon=2 \delta^{3 / 4}\right)$ мы получаем для $\delta<\|h\|$, что

$$
\|u-d(u)\|<\sqrt{N} 2 \delta^{3 / 4} \leqslant 2\|h\|^{1 / 2} \delta^{-1 / 2} 2 \delta^{3 / 4}=4\|h\|^{1 / 2} \sqrt[4]{\delta}
$$

что завершает доказательство леммы 2.3.

Определим путь $u(t)$ формулой

$$
u(t)=(u-d(u))(1-t)+d(u) .
$$

Тогда $u(0)=u, u(1)=d(u)$, и для каждого $t \in[0,1]$ согласно лемме 2.3 мы имеем $\operatorname{dist}(u(t), U(A))<4\|h\|^{1 / 2} \sqrt[4]{\delta}$. Оценим норму коммутатора:

$$
\begin{aligned}
\|u(t) \bar{h}-\bar{h} u(t)\| & \leqslant\|u \bar{h}-\bar{h} u\|+\|(u-d(u)) \bar{h}-\bar{h}(u-d(u))\| \\
& <2 \delta+2\|h\|\|u-d(u)\|<2 \delta+2\|h\| 4\|h\|^{1 / 2} \sqrt[4]{\delta}
\end{aligned}
$$

Второй шаг гомотопии. Нам нужно соединить матрицу $d(u)$ с диагональной матрицей. Для этого нам необходима следуюшая 
ЛЕмма 2.4 (приведение к почти верхнетреугольному виду). Пусть

$$
a=\left(\begin{array}{ll}
a_{11} & a_{12} \\
a_{21} & a_{22}
\end{array}\right)
$$

есть матрица в $q_{j_{1}} A \oplus q_{j_{2}} A$. Тогда для любого $\varepsilon>0$ существует унитарньий nуть $v(t)$ такой, что $v(0)=1$,

$$
v(1) \cdot a=\left(\begin{array}{cc}
a_{11}^{\prime} & a_{12}^{\prime} \\
a_{21}^{\prime} & a_{22}^{\prime}
\end{array}\right)
$$

əде $\left\|a_{21}^{\prime}\right\|<\varepsilon$.

ДокАЗАТЕльСТво. По предположению $\operatorname{tsr}(A)=1$, поэтому для любого $\varepsilon>0$ можно найти обратимый элемент $\bar{a}_{11}$ такой, что $\left\|\bar{a}_{11}-a_{11}\right\|<\varepsilon$. Положим $\alpha=a_{21}\left(\bar{a}_{11}\right)^{-1}$

$$
v(t)=\left(\begin{array}{cc}
\left(1+t^{2} \alpha^{*} \alpha\right)^{-1 / 2} & \left(1+t^{2} \alpha^{*} \alpha\right)^{-1 / 2} \cdot t \alpha^{*} \\
-\left(1+t^{2} \alpha \alpha^{*}\right)^{-1 / 2} \cdot t \alpha & \left(1+t^{2} \alpha \alpha^{*}\right)^{-1 / 2}
\end{array}\right) .
$$

Легко видеть, что элемент $v(1)$ унитарен. Оценим матричный элемент $a_{21}^{\prime}$ в произведении $v(1) \cdot a$

$$
\begin{aligned}
\left\|a_{21}^{\prime}\right\| & =\left\|-\left(1+\alpha \alpha^{*}\right)^{-1 / 2} \alpha a_{11}+\left(1+\alpha \alpha^{*}\right)^{-1 / 2} a_{21}\right\| \\
& =\left\|\left(1+\alpha \alpha^{*}\right)^{-1 / 2}\left(\alpha a_{11}-\alpha \bar{a}_{11}\right)\right\| \leqslant\left\|\left(1+\alpha \alpha^{*}\right)^{-1 / 2} \alpha\right\| \cdot\left\|a_{11}-\bar{a}_{11}\right\| \\
& <\varepsilon \cdot\left\|\left(1+\alpha \alpha^{*}\right)^{-1 / 2} \alpha\right\|=\varepsilon \cdot\|f(\beta)\|^{1 / 2} \leqslant \varepsilon,
\end{aligned}
$$

где $\beta=\alpha \alpha^{*}$ и $f(\lambda)=\lambda /(1+\lambda) \leqslant 1$.

Рассмотрим элемент $u_{21}$ матрицы $u$. Если отрезки $\Delta_{1}$ и $\Delta_{2}$ отделены друг от друга, то элемент $u_{21}$ уже достаточно мал, а элемент $(d(u))_{21}$ уже нулевой. Положим в этом случае $v_{1}=1$. Если эти отрезки имеют обшую граничную точку, то по лемме 2.4 можно найти в модуле $q_{1} A \oplus q_{2} A$ унитарньй путь $v_{1}(t)$ такой, что $v_{1}(0)=1$,

$$
v_{1}(1)=\left(\begin{array}{cc}
v_{11}^{(1)} & v_{12}^{(1)} \\
v_{21}^{(1)} & v_{22}^{(1)}
\end{array}\right)
$$

И

$$
\left(\begin{array}{cccc}
v_{11}^{(1)} & v_{12}^{(1)} & \vdots & \\
v_{21}^{(1)} & v_{22}^{(1)} & \vdots & \\
\cdots & \ldots & \ldots & \ldots \\
& & \vdots & E
\end{array}\right) \cdot\left(\begin{array}{cccc}
u_{11} & u_{12} & & \\
u_{21} & u_{22} & u_{23} & \\
& \ldots & \ldots & \ldots \\
& \ldots & \ldots
\end{array}\right)=\left(\begin{array}{cccc}
u_{11}^{(1)} & u_{12}^{(1)} & u_{13}^{(1)} & \\
u_{21}^{(1)} & u_{22}^{(1)} & u_{23}^{(1)} & \\
& \ldots & \ldots & \ldots \\
& & \ldots & \ldots
\end{array}\right),
$$

где $\left\|u_{21}^{(1)}\right\|<\varepsilon$. Здесь $E$ обозначает единичную матрицу, а пустые места - нули. Заметим, что третья и следующие строки матрицы $u$ не меняются. Мы получаем путь $u(t)=v_{1}(t) d(u)$, и поскольку $v_{1}(t)$ унитарен,

$$
\operatorname{dist}(u(t), U)<4\|h\|^{1 / 2} \sqrt[4]{\delta}
$$


Так как в лемме 2.4 число $\varepsilon>0$ можно выбирать произвольньм образом, оценка (2.6) остается верной, если мы заменим элемент $u_{21}^{(1)}$ на нуль.

Обратимся теперь к элементу $u_{32}=u_{32}^{(1)}$. Если отрезки $\Delta_{2}$ и $\Delta_{3}$ отделены друг от друга, то этот элемент уже достаточно мал. Положим тогда $v_{2}=1$. В противном случае найдем унитарный путь $v_{2}(t), t \in[1,2]$, такой, что $v_{2}(1)=1$, а матрица

$$
v_{2}(2)=\left(\begin{array}{cc}
v_{22}^{(2)} & v_{23}^{(2)} \\
v_{32}^{(2)} & v_{33}^{(2)}
\end{array}\right)
$$

в модуле $q_{2} A \oplus q_{3} A$ такова, что

$$
\begin{aligned}
& \left(\begin{array}{cccccc}
1 & & & & \\
& v_{22}^{(2)} & v_{23}^{(2)} & \vdots & \\
& v_{32}^{(2)} & v_{33}^{(2)} & \vdots & \\
\ldots & \ldots & \ldots & \ldots \\
& & & \vdots & E
\end{array}\right) \cdot\left(\begin{array}{cccccc}
u_{11}^{(1)} & u_{12}^{(1)} & u_{13}^{(1)} & & \\
& u_{22}^{(1)} & u_{23}^{(1)} & & \\
& u_{32}^{(1)} & u_{33}^{(1)} & u_{34}^{(1)} & \\
& & \ldots & \ldots & \ldots \\
& & & \ldots & \ldots
\end{array}\right) \\
& =\left(\begin{array}{ccccc}
u_{11}^{(2)} & u_{12}^{(2)} & u_{13}^{(2)} & & \\
& u_{22}^{(2)} & u_{23}^{(2)} & u_{24}^{(2)} & \\
& u_{32}^{(2)} & u_{33}^{(2)} & u_{34}^{(2)} & \\
& & \ldots & \ldots & \ldots \\
& & & \ldots & \ldots
\end{array}\right)
\end{aligned}
$$

где $\left\|u_{32}^{(2)}\right\|<\varepsilon$. Опять мы можем заменить элемент $u_{32}^{(2)}$ нулем. Повторяя эту процедуру, получаем унитарный путь

$$
v(t)=v_{m-1}(t) \cdot \ldots \cdot v_{2}(t) \cdot v_{1}(t), \quad t \in[0, m-1]
$$

соединяющий единицу с унитарньп элементом

$$
v=v(m-1)=v_{m-1}(m-1) \cdot \ldots \cdot v_{2}(2) \cdot v_{1}(1) .
$$

Обозначим $v(t) \cdot d(u)$ через $\bar{u}(t)$, а $v \cdot d(u)$ - через $\bar{u}$. Заметим, что

$$
\operatorname{dist}(\bar{u}(t), U)<4\|h\|^{1 / 2} \sqrt[4]{\delta}
$$

для всех $t$, так как умножение на унитарный элемент не меняет расстояния до унитарной группы. Отсюда получается оценка $\left\|\bar{u}_{i j}(t)\right\|<4\|h\|^{1 / 2} \sqrt[4]{\delta}$ для всех $i, j$. Заметим также, что для всех $t$ путь $\bar{u}(t)$ лежит в четырех диагональных матрицах вида

$$
\bar{u}(t)=\left(\begin{array}{cccccc}
\bar{u}_{11} & \bar{u}_{12} & \bar{u}_{13} & & & \\
\bar{u}_{21} & \bar{u}_{22} & \bar{u}_{23} & \bar{u}_{24} & & \\
& \bar{u}_{32} & \bar{u}_{33} & \bar{u}_{34} & \bar{u}_{35} & \\
& & \ldots & \ldots & \ldots & \ldots \\
& & & \ldots & \ldots & \ldots \\
& & & & \ldots & \ldots
\end{array}\right)
$$


поэтому легко оценить норму коммутатора вдоль этого пути. Для этого удобнее работать с оператором $h^{\prime}=\sum \mu_{k} q_{k},\left\|h-h^{\prime}\right\|<\frac{1}{2} \sqrt[4]{\delta}$. Тогда

$$
\begin{aligned}
\|\bar{u}(t) h-h \bar{u}(t)\| & <\left\|\left[\bar{u}(t), h^{\prime}\right]\right\|+\sqrt[4]{\delta} \\
& \leqslant\left\|\left[d_{-1}(\bar{u}(t)), h^{\prime}\right]\right\|+\left\|\left[d_{1}(\bar{u}(t)), h^{\prime}\right]\right\|+\left\|\left[d_{2}(\bar{u}(t)), h^{\prime}\right]\right\|+\sqrt[4]{\delta} \\
& \leqslant \sup _{i, j, k}\left\|\bar{u}_{i j}(t)\right\|\left(2\left|\mu_{k}-\mu_{k+1}\right|+\left|\mu_{k}-\mu_{k+2}\right|\right)+\sqrt[4]{\delta}<6 \sqrt[4]{\delta}
\end{aligned}
$$

для достаточно малых $\delta$. Окончательная матрища $\bar{u}$ является трех диагональной и верхнетреугольной:

$$
\bar{u}=\left(\begin{array}{ccccc}
\bar{u}_{11} & \bar{u}_{12} & \bar{u}_{13} & & \\
& \bar{u}_{22} & \bar{u}_{23} & \bar{u}_{24} & \\
& & \bar{u}_{33} & \bar{u}_{34} & \bar{u}_{35} \\
& & & \ldots & \ldots \\
& & & & \ldots
\end{array}\right) .
$$

Третий шаг гомотопии. Из (2.7) следует, что матрища $\bar{u}$ обратима и сушествует унитарный элемент $w$ такой, что

$$
\|\bar{u}-w\|<4\|h\|^{1 / 2} \sqrt[4]{\delta} .
$$

Поэтому, если $\delta$ достаточно мало,

$$
\left\|(\bar{u})^{-1}-w^{*}\right\|<6\|h\|^{1 / 2} \sqrt[4]{\delta}
$$

Следовательно, получаем оценку

$$
\left\|(\bar{u})^{-1}-(\bar{u})^{*}\right\|<10\|h\|^{1 / 2} \sqrt[4]{\delta}
$$

и, значит,

$$
\left\|d_{1}(\bar{u})\right\|<10\|h\|^{1 / 2} \sqrt[4]{\delta}, \quad\left\|d_{2}(\bar{u})\right\|<10\|h\|^{1 / 2} \sqrt[4]{\delta}
$$

где $d_{k}-k$-я диаголаль. Поэтому

$$
\operatorname{dist}\left(d_{0}(\bar{u}), U\right)<\operatorname{dist}(\bar{u}, U)+\left\|d_{1}(\bar{u})\right\|+\left\|d_{2}(\bar{u})\right\|<24\|h\|^{1 / 2} \sqrt[4]{\delta} .
$$

Соединим матрицы $\bar{u}$ и $d_{0}(\bar{u})$ линейньм путем $\overline{\bar{u}}(t)$. Тогда вдоль этого пути

$$
\operatorname{dist}(\overline{\bar{u}}(t), U)<24\|h\|^{1 / 2} \sqrt[4]{\delta},
$$

а из (2.8) следует, что

$$
\|\overline{\bar{u}}(t) h-h \overline{\bar{u}}(t)\|<6\|h\|^{1 / 2} \sqrt[4]{\delta} .
$$

Четвертый шаг гомотопии. Из (2.9) следует, что для достаточно малых $\delta$ диагональная матрица $d_{0}(\bar{u})$ состоит из обратимых элементов, близких к унитарным, и из (2.10) следует, что для каждого $\bar{u}_{i i}$ можно найти унитарный элемент $w_{i}$ такой, что $\left\|\bar{u}_{i i}-w_{i}\right\|<24\|h\|^{1 / 2} \sqrt[4]{\delta}$. Возьмем линейный путь, соединяющий матрицы $\bar{u}$ и $\operatorname{diag}\left(\left\{w_{i}\right\}\right)$. Этот путь также лежит близи унитарной группы $U$. Соединим затем матрицу $\operatorname{diag}\left(\left\{w_{i}\right\}\right)$ с единицей. Последний путь лежит в $U$. Пусть $\widetilde{u}(t)$ есть путь, 
построенный на четвертом шаге гомотопии. Заметим, что он лежит в диагональных матрицах, поэтому норма коммутатора вдоль этого пути мала

$$
\|\widetilde{u}(t) h-h \widetilde{u}(t)\| \leqslant\left\|\widetilde{u}(t) h^{\prime}-h^{\prime} \widetilde{u}(t)\right\|+\sqrt[4]{\delta}=\sqrt[4]{\delta}
$$

Рассмотрим теперь все четыре шага построенной гомотопии. Вдоль всего пути $u^{\prime}(t)$, соединяющего $u$ с единицей, верны оценки

$$
\operatorname{dist}\left(u^{\prime}(t), U\right)<24\|h\|^{1 / 2} \sqrt[4]{\delta}
$$

и

$$
\left\|u^{\prime}(t) h-h u^{\prime}(t)\right\|<6 \sqrt[4]{\delta}
$$

Поэтому существует унитарный путь $u(t)$, соединяющий $u$ с единицей, такой, что

$$
\left\|u(t)-u^{\prime}(t)\right\|<48\|h\|^{1 / 2} \sqrt[4]{\delta}
$$

значит, мы получаем оценку

$$
\begin{aligned}
\|u(t) h-h u(t)\| & <\left\|u^{\prime}(t) h-h u^{\prime}(t)\right\|+2\|h\|\left\|u(t)-u^{\prime}(t)\right\| \\
& <6 \sqrt[4]{\delta}+96\|h\|^{3 / 2} \sqrt[4]{\delta}=C \sqrt[4]{\delta}
\end{aligned}
$$

Предложение 2.1 доказано.

Заметим, что класс $C^{*}$-алгебр, удовлетворяюших условиям предложения 2.1, включает конечные $W^{*}$-алгебры и, в частности, матричные алгебры. Константа $C$ не является наилучшей. Однако степень $\frac{1}{4}$ для $\delta$, как нам кажется, не может быть увеличена. Поскольку норма коммутатора не меняется при замене $h$ на $h+\lambda$, $\lambda \in \mathbb{R}$, то константа $C$ на самом деле зависит не от нормы $h$, а от длины его спектра.

Заметим также, что тем же способом можно доказать аналогичный результат, если заменить самосопряженный оператор $h$ на унитарный оператор $v$ с достаточно большой лакуной в спектре. Последнее предположение сушественно, так как в противном случае существует препятствие [2].

ПРЕДЛОЖЕНИЕ 2.5. Пусть $A$ есть $C^{*}$-алгебра, удовлетворяющая условиЯМ्M:

i) $R R(A)=0 u \operatorname{tsr}(A)=1$;

ii) для любого проектора $p \in A$ унитарная группа $C^{*}$-алгебры $р А р$ связна. Пусть $u, v \in A$ суть унитарные әлементь такие, что $\|u v-v u\|<\delta$. Предположим, что единичная окружность содержит дугу длины $d$, свободную от точек спектра $v$. Тогда существуют константы $C$ и $\delta_{0}>0$, зависящие только от $d$, и унитарньй путь $u(t)$, соединяющий и с единицей, такой, что nри $\delta<\delta_{0}$ для всеx $t \quad\|u(t) v-v u(t)\|<C \sqrt[4]{\delta}$. 


\section{§3. Диагонализация операторов над некоторыми непрерывными полями $C^{*}$-алгебр}

Tеорема 3.1. Пусть $(A(x), X, \mathscr{A})$ есть непрерьвное поле $C^{*}$-алгебр со следом над отрезком или окружностью. Пусть $(B(x), X, \mathscr{B})$ есть соответствующее непрерывное поле $W^{*}$-алгебр, а $\mathbf{A}$ - соответствующая $W^{*}$-алгебра. Предположим, что для каждой точки $x \in X C^{*}$-алгебра $A(x)$ обладает следующими свойствами:

i) $R R(A(x))=0 u \operatorname{tsr}(A(x))=1$;

ii) для любого проектора $p \in A(x)$ унитарная группа $C^{*}$-алгебры $p A(x) p$ связна;

iii) след $\tau_{x}$ на $A(x)$ является конечным и точным;

iv) гомоморфизм $K_{0}(A(x)) \rightarrow K_{0}(B(x))$, индуцированный вложением $A(x) \subset B(x)=L^{\infty}(A(x))$, является мономорфизмом, и $K_{0}(A(x))$ является подрешеткой в $K_{0}(B(x))$.

Тогда $C^{*}$-алгебра $\mathscr{A} \in \mathbf{A}$ обладает свойством слабой диагонализации.

ДокАЗАТЕЛЬСТво основано на следующих леммах. Через $M_{n}$ мы обозначаем алгебру $(n \times n)$-матриц.

Лемма 3.2. Пусть $K_{1}(x), K_{2}(x)$ суть два непрерывных поля самосопряженных операторов с конечным спектром в алгебре $M_{n} \otimes \mathscr{A}$ таких, что $K_{k}$ унитарно эквивалентны $\operatorname{diag}\left(\left\{\lambda_{i}^{(k)}\right\}\right), k=1,2$. Если $\left\|K_{1}(x)-K_{2}(x)\right\|<\varepsilon$, то для любого $x_{0} \in X$ существуют замкнутая окрестность $W$ точки $x_{0} u$ непрерьвнье унитарные поля $u_{i}(x)$ в $W$ такие, что

$$
\left\|\lambda_{i}^{(1)}(x)-u_{i}^{*}(x) \lambda_{i}^{(2)}(x) u_{i}(x)\right\|<\varepsilon .
$$

ДокАЗАТЕЛЬСТво. По предположению мы можем записать операторы $K_{k}(x)$ в виде

$$
K_{1}(x)=\sum \alpha_{m} p_{m}(x), \quad K_{2}(x)=\sum \beta_{j} q_{j}(x),
$$

где $p_{m}(x), q_{j}(x)$ - спектральные проекторы, и собственные значения упорядочены. Так как $K_{0}\left(A\left(x_{0}\right)\right) \subset K_{0}\left(B\left(x_{0}\right)\right)$ является подрешеткой по предположению, мы можем найти проекторы $r_{l}\left(x_{0}\right) \in A\left(x_{0}\right)$ такие, что $\sum_{l} r_{l}\left(x_{0}\right)=1 \in M_{n} \otimes A\left(x_{0}\right)$, и каждый из проекторов $p_{m}\left(x_{0}\right), q_{j}\left(x_{0}\right)$, а также проекторы из $M_{n} \otimes 1$ унитарно эквивалентны суммам некоторых $r_{l}\left(x_{0}\right)$. Перенумеровав собственные значения $K_{k}\left(x_{0}\right)$ и допуская повторение собственных значений, мы можем записать

$$
K_{1}\left(x_{0}\right)=\sum \alpha_{l} r_{l}\left(x_{0}\right), \quad K_{2}\left(x_{0}\right)=\sum \beta_{l} r_{l}^{\prime}\left(x_{0}\right), \quad r_{l}\left(x_{0}\right) \sim r_{l}^{\prime}\left(x_{0}\right) .
$$

Так как в $W^{*}$-алгебре $M_{n} \otimes B\left(x_{0}\right)=M_{n} \otimes L^{\infty}\left(A\left(x_{0}\right)\right)$ выполнена оценка $\left\|K_{1}\left(x_{0}\right)-K_{2}\left(x_{0}\right)\right\|<\varepsilon$, то (cp. [21]) для всех $l$ получаем

$$
\left|\alpha_{l}-\beta_{l}\right|<\varepsilon .
$$

Разделим множество проекторов $\left\{r_{l}\left(x_{0}\right)\right\}$ на $s$ групा

$$
\left\{r_{1}\left(x_{0}\right), \ldots, r_{l_{1}}\left(x_{0}\right)\right\}, \ldots,\left\{r_{l_{s-1}+1}\left(x_{0}\right), \ldots, r_{l_{s}}\left(x_{0}\right)\right\}
$$


так, чтобы сумма проекторов в каждой группе была бы унитарно эквивалентна одномерному проектору в $M_{n} \otimes 1$. Тогда каждой группе соответствует “собственное значение" $\lambda_{i}^{(k)}\left(x_{0}\right)$ :

$$
\begin{aligned}
& \lambda^{(1)}\left(x_{0}\right)=\alpha_{l_{i-1}+1} r_{l_{i-1}+1}\left(x_{0}\right)+\cdots+\alpha_{l_{i}} r_{l_{i}}\left(x_{0}\right), \\
& \lambda^{(2)}\left(x_{0}\right)=\beta_{l_{i-1}+1} r_{l_{i-1}+1}^{\prime}\left(x_{0}\right)+\cdots+\beta_{l_{i}} r_{l_{i}}^{\prime}\left(x_{0}\right) .
\end{aligned}
$$

Выберем унитарные элементы $u_{i}\left(x_{0}\right) \in A\left(x_{0}\right)$ такие, что

$$
r_{l}^{\prime}\left(x_{0}\right)=u_{i}^{*}\left(x_{0}\right) r_{l}\left(x_{0}\right) u_{i}\left(x_{0}\right), \quad l_{i-1}+1 \leqslant l \leqslant l_{i} .
$$

Тогда оценка (3.1) следует из (3.2).

Лемма 3.3. Пусть $D=\left[x_{k}, x_{k+1}\right]$ есть отрезок в $X, \lambda(x),\left.K_{2}^{\prime}(x) \in \mathscr{A}\right|_{D}{ }_{-}$ два непрерывных поля самосопряжсенных операторов с конечным спектром на $D$ таких, что $\left\|\lambda(x)-\mu^{\prime}(x)\right\|<\varepsilon$. Пусть $\mu \in A\left(x_{k}\right)$ есть самосопряженный оператор с конечным спектром такой, что существует унитарный әлемент $u \in A\left(x_{k}\right)$,

$$
\left\|\mu-u^{*} \mu^{\prime}\left(x_{k}\right) u\right\|<\delta, \quad\left\|\lambda\left(x_{k}\right)-\mu\right\|<\varepsilon .
$$

Тогда для достаточно малых $\delta$ и є существует кусочно-непрерывное унитарное поле $и(x)$ такое, что

i) $u\left(x_{k}\right)=u, u\left(x_{k+1}\right)=1$;

ii) на $D$ имеет место оценка

$$
\left\|u^{*}(x) \mu^{\prime}(x) u(x)-\lambda(x)\right\|<C \sqrt[4]{2 \varepsilon+\delta}
$$

әде $C$-константа, зависящая только от нормы $\lambda(x)$.

ДокАЗАТЕЛЬство. Пусть $u_{t}, 0 \leqslant t \leqslant 1,-$ путь, соединяющий $u$ с единицей, в унитарной группе алгебры $A\left(x_{k}\right)$. По предположению $\left\|\mu-\mu^{\prime}\left(x_{k}\right)\right\|<2 \varepsilon$, следовательно,

$$
\left\|\mu^{\prime}\left(x_{k}\right)-u_{t}^{*} \mu^{\prime}\left(x_{k}\right) u_{t}\right\|<2 \varepsilon+\delta .
$$

Тогда по предложению 2.1 найдутся константа $C$, зависящая только от нормы $\lambda\left(x_{k}\right)$ (или $\mu^{\prime}\left(x_{k}\right)$, так как они близки), и путь $u_{t}$, соединяющий $u$ с единицей, такие, что

$$
\left\|u_{t}^{*} \mu^{\prime}\left(x_{k}\right) u_{t}-\lambda\left(x_{k}\right)\right\|<C \sqrt[4]{2 \varepsilon+\delta}
$$

Из непрерывности следует, что существует точка $x_{k+1}^{\prime} \in\left(x_{k}, x_{k+1}^{\prime}\right)$ такая, что над отрезком $\left[x_{k}, x_{k+1}\right]$ сушествует унитарное непрерывное поле $u_{t}(x)$, продолжающее $u_{t}$, и для всех $x \in\left[x_{k}, x_{k+1}^{\prime}\right]$ по-прежнему верна оценка

$$
\left\|u_{t}^{*}(x) \mu^{\prime}(x) u_{t}(x)-\lambda(x)\right\|<C \sqrt[4]{2 \varepsilon+\delta} .
$$

Определим унитарное поле $u(x)$ формулой

$$
u(x)= \begin{cases}u_{t}(x), t=\frac{x_{k+1}^{\prime}-x}{x_{k+1}^{\prime}-x_{k}}, & \text { если } x \in\left[x_{k}, x_{k+1}^{\prime}\right], \\ 1, & \text { если } x \in\left[x_{k+1}^{\prime}, x_{k+1}\right] .\end{cases}
$$

Это - унитарное непрерывное поле, и на интервале $\left[x_{k}, x_{k+1}^{\prime}\right]$ выполняется оценка (3.3). С другой стороны, когда $x \geqslant x_{k+1}, u(x)=1$, поэтому там эта оценка также выполнена. 
Лемма 3.4. Пусть $K(x)$ есть самосопряжсенный оператор в модуле $L_{n}(\mathscr{A})$, $K(x) \in M_{n} \otimes \mathscr{A}$. Тогда для любого $\varepsilon>0$ существует кусочно-непрерывное поле операторов с дискретным спектром $K^{\prime}(x)$, локально лежсащее в $M_{n} \otimes \mathscr{A}$, такое, что $\left\|K^{\prime}(x)-K(x)\right\|<\varepsilon$, оператор $K^{\prime}(x)$ диагонализируем в $L_{n} \otimes \mathbf{A} c$ кусочно-непрерывными “собственными значениями" $\lambda_{i}(x), \operatorname{dist}\left(\lambda_{i}(x), \mathscr{A}\right)<2 \varepsilon$.

ДокаЗАТЕЛьство. Зафиксируем $\varepsilon>0$ и выберем точку $x_{0} \in X$. Поскольку $R R\left(A\left(x_{0}\right)\right)=0$, мы можем найти оператор $K^{\prime}\left(x_{0}\right) \in M_{n} \otimes A\left(x_{0}\right)$ с конечньм спектром, $K^{\prime}\left(x_{0}\right)=\sum_{m} \alpha_{m} p_{m}\left(x_{0}\right)$ (где $p_{m}\left(x_{0}\right)$ - его спектральные проекторы) такой, что $\left\|K^{\prime}\left(x_{0}\right)-K\left(x_{0}\right)\right\|<\varepsilon / 2$. Сушествует окрестность точки $x_{0}$ такая, что проекторы $p_{m}\left(x_{0}\right)$ могут быть продолжены непрерывным образом до проекторов $p_{m}(x)$ в этой окрестности. Положим $K^{\prime}(x)=\sum_{m} \alpha_{m} p_{m}(x)$. Тогда можно найти меньшую окрестность точки $x_{0}$, в которой

$$
\left\|K^{\prime}(x)-K(x)\right\|<\varepsilon
$$

Итак, для каждой точки в $X$ сушествуют окрестность $W$ и непрерывное поле на $W$ такие, что выполнено неравенство (3.4). Взяв конечное покрытие $X$ такими окрестностями, можно получить разбиение $X$ на отрезки $\Delta_{k}$ и кусочно-непрерывное поле (непрерьвное на каждом отрезке) $K^{\prime}(x)=\left\{K_{k}^{\prime}(x)\right\},\left.K_{k}^{\prime}(x) \in M_{n} \otimes \mathscr{A}\right|_{D_{k}}$ такое, что $\operatorname{dist}\left(K^{\prime}(x), M_{n} \otimes \mathscr{A}\right)<2 \varepsilon$. Диагонализируем операторное поле $K^{\prime}(x)$ на каждом отрезке $D_{k}, K^{\prime}(x)=\operatorname{diag}\left(\left\{\lambda_{i}(x)\right\}\right)$. Тогда поля $\lambda_{i}(x)=\left\{\lambda_{i}^{(k)}(x)\right\}, \lambda_{i}^{(k)}(x) \in$ $\left.\mathscr{A}\right|_{D_{k}}$, являются кусочно-непрерьвньми, и поскольку $\left\|K_{k-1}^{\prime}\left(x_{k}\right)-K_{k}^{\prime}\left(x_{k}\right)\right\|<2 \varepsilon$, то, используя леммы 3.2 и 3.3 , мы можем заменить диагональные элементы $\lambda_{i}(x)$ на каждом интервале на унитарно эквивалентные так, чтобы выполнялась оценка

$$
\operatorname{dist}\left(\lambda_{i}(x), \mathscr{A}\right)=\sup _{k}\left\|\lambda_{i}^{(k-1)}\left(x_{k}\right)-\lambda_{i}^{(k)}\left(x_{k}\right)\right\|<2 \varepsilon
$$

ЛЕмма 3.5. Пусть $K_{1}(x), K_{2}(x) \in M_{n} \otimes \mathbf{A}$ суть кусочно-непрерьвные поля операторов с конечным спектром, $\left\|K_{1}(x)-K_{2}(x)\right\|<\varepsilon, K_{1}(x) \sim \operatorname{diag}\left(\left\{\lambda_{i}(x)\right\}\right)$, $K_{2}(x) \sim \operatorname{diag}\left(\left\{\mu_{i}(x)\right\}\right)$. Пусть, далее, их диагональные әлементы $\lambda_{i}(x)$ и $\mu_{i}(x)$ кусочно-непрерывны $u \operatorname{dist}\left(\mu_{i}(x), \mathscr{A}\right)<\delta$. Тогда существуют кусочно-непрерывные унитарные поля $u_{i}(x)$, кусочно-непрерывные поля $\mu_{i}^{\prime}(x)$ и кусочно-непрерьвное поле операторов $K_{2}^{\prime}(x) \sim \operatorname{diag}\left(\left\{\mu_{i}^{\prime}(x)\right\}\right)$ такие, что

i) $\left\|K_{2}^{\prime}(x)-K_{2}(x)\right\|<\delta$;

ii) $\left\|u_{i}^{*}(x) \mu_{i}^{\prime}(x) u_{i}(x)-\mu_{i}(x)\right\|<\delta$;

iii) $\left\|\lambda_{i}(x)-\mu_{i}^{\prime}(x)\right\|<C \sqrt[4]{2 \varepsilon+\delta}$;

iv) $\operatorname{dist}\left(\mu_{i}^{\prime}(x), \mathscr{A}\right)<\delta$.

ДокАЗАТЕЛЬСтво. Пусть $x_{0} \in X$ есть точка непрерывности для $K_{2}(x), D \supset x_{0}$ - интервал в $X$. По лемме 3.2 мы можем диагонализировать оператор $K_{2}\left(x_{0}\right)$ так, что его “собственные значения" $\widetilde{\mu}_{i}\left(x_{0}\right)$ будут удовлетворять неравенству

$$
\left\|\widetilde{\mu}_{i}\left(x_{0}\right)-\lambda\left(x_{0}\right)\right\|<\varepsilon .
$$


Продолжим эти "собственные значения" произвольным образом до непрерывных полей $\widetilde{\mu}_{i}(x)$. Тогда в некоторой окрестности точки $x_{0}$ по-прежнему выполнено неравенство

$$
\left\|\widetilde{\mu}_{i}(x)-\lambda_{i}(x)\right\|<\varepsilon .
$$

По предположению существуют унитарные элементы $w_{i} \in A\left(x_{0}\right)$ такие, что $w_{i}^{*} \widetilde{\mu}\left(x_{0}\right) w_{i}=\mu_{i}\left(x_{0}\right)$. Возьмем унитарные продолжения $w_{i}(x)$ элементов $w_{i}$, $w_{i}\left(x_{0}\right)=w_{i}$, и выберем окрестность точки $x_{0}$ так, чтобы оценка

$$
\left\|w_{i}^{*}(x) \widetilde{\mu}_{i}(x) w_{i}(x)-\mu_{i}(x)\right\|<\delta
$$

вьполнялась в этой окрестности. Так мы получим разбиение $X$ на отрезки $D_{k}=\left[x_{k}, x_{k+1}\right]$, а также кусочно-непрерьвные поля $\tilde{\mu}_{i}(x),\left.\widetilde{\mu}_{i}(x)\right|_{D_{k}}=\widetilde{\mu}_{i}^{(k)}(x)$, $\left.\tilde{\mu}_{i}^{(k)}(x) \in \mathscr{A}\right|_{D_{k}}$, и кусочно-непрерывные унитарные поля $w_{i}(x)=\left\{w_{i, k}(x)\right\}$, $\left.w_{i, k}(x) \in \mathscr{A}\right|_{D_{k}}$, такие, что на $D_{k}$ выполнены оценки

$$
\left\|\widetilde{\mu}_{i}^{(k)}(x)-\lambda_{i}(x)\right\|<\varepsilon
$$

$$
\left\|w_{i, k}^{*}(x) \widetilde{\mu}_{i}^{(k)}(x) w_{i, k}(x)-\mu_{i}(x)\right\|<\frac{\delta}{2} .
$$

Сушествует также кусочно-непрерывное поле операторов $\widetilde{K}_{2}(x)$, унитарно эквивалентное оператору $\operatorname{diag}\left(\left\{\widetilde{\mu}_{i}(x)\right\}\right)$, и такое, что

$$
\left\|\widetilde{K}_{2}(x)-K_{2}(x)\right\|<\frac{\delta}{2} .
$$

Из (3.6) следует, что в точке $x_{k}$

$$
\left\|w_{i, k-1}^{*}\left(x_{k}\right) \widetilde{\mu}_{i}^{(k-1)}\left(x_{k}\right) w_{i, k-1}\left(x_{k}\right)-w_{i, k}^{*}\left(x_{k}\right) \widetilde{\mu}_{i}^{(k)}\left(x_{k}\right) w_{i, k}\left(x_{k}\right)\right\|<\delta,
$$

а из (3.7) следует существование таких унитарных элементов $v_{i, k}\left(x_{k}\right) \in A\left(x_{k}\right)$, что

$$
\left\|v_{i, k}^{*}\left(x_{k}\right) \widetilde{\mu}_{i}^{(k-1)}\left(x_{k}\right) v_{i, k}\left(x_{k}\right)-\widetilde{\mu}_{i}^{(k)}\left(x_{k}\right)\right\|<\delta .
$$

По лемме 3.3 мы можем найти такие продолжения унитарных элементов $v_{i, k}\left(x_{k}\right)$ в некоторую окрестность точки $x_{k}$, для которых согласно (3.5) имеет место оценка

$$
\left\|v_{i, k}^{*}(x) \widetilde{\mu}_{i}^{(k)}(x) v_{i, k}(x)-\lambda_{i}(x)\right\|<C \sqrt[4]{2 \varepsilon+\delta} .
$$

Положим

$$
\bar{\mu}_{i}^{(k)}(x)=v_{i, k}(x) \widetilde{\mu}_{i}^{(k)}(x) v_{i, k}^{*}(x) .
$$

Тогда

$$
\left\|\bar{\mu}_{i}^{(k)}(x)-\widetilde{\mu}_{i}^{(k-1)}(x)\right\|<\delta
$$

$$
\left\|\bar{\mu}_{i}^{(k)}(x)-\lambda_{i}(x)\right\|<C \sqrt[4]{2 \varepsilon+\delta} .
$$


Действуя по индукции и переходя от $D_{k-1}$ к $D_{k}$, мы заменяемполя $\widetilde{\mu}_{i}^{(k)}(x)$ на $\bar{\mu}_{i}^{(k)}(x)$ на каждом $D_{k}$ и получаем новые кусочно-непрерывные поля $\mu_{i}^{\prime}(x)=\left\{\bar{\mu}_{i}^{(k)}(x)\right\}$ такие, что сушествуют кусочно-непрерывные поля унитарных элементов $u_{i}(x)$, для которых

$$
\left\|u_{i}^{*}(x) \mu_{i}^{\prime}(x) u_{i}(x)-\mu_{i}(x)\right\|<\delta .
$$

Положив $K_{2}^{\prime}(x)=\operatorname{diag}\left(\left\{u_{i}(x) \mu_{i}^{\prime}(x) u_{i}^{*}(x)\right\}\right)$, получаем утверждение i), а оценка $\operatorname{dist}\left(\mu_{i}^{\prime}(x), \mathscr{A}\right)<\delta$ следует из $(3.8)$.

Пусть теперь $K(x)$ есть строго положительньй компактный оператор в модуле $H_{\mathscr{A}}$. Возьмем последовательность $\varepsilon_{m}>0$, сходящуюся к нулю. Из компактности $K(x)$ по лемме 3.4 мы можем найти последовательность кусочно-непрерывных полей диагонализируемых операторов конечного ранга $K_{m}(x) \in M_{n_{m}} \otimes \mathbf{A}$ c "собственными значениями" $\lambda_{i, m}(x) \in \mathscr{A}$ такими, что

i) $\left\|K_{m}(x)-K(x)\right\|<\varepsilon_{m}$;

ii) $K_{m}(x) \sim \operatorname{diag}\left(\left\{\lambda_{i, m}(x)\right\}\right)$, где $\operatorname{dist}\left(\lambda_{i, m}(x), \mathscr{A}\right)<\varepsilon_{m}$.

Поскольку

$$
\left\|K_{1}(x)-K_{2}(x)\right\| \leqslant\left\|K_{1}(x)-K(x)\right\|+\left\|K(x)-K_{2}(x)\right\|<\varepsilon_{1}+\varepsilon_{2},
$$

то по лемме 3.5 можно найти кусочно-непрерывный оператор $K_{2}^{\prime}(x) \sim$ $\operatorname{diag}\left(\left\{\lambda_{i, 2}^{\prime}(x)\right\}\right)$ такой, что

$$
\begin{gathered}
\left\|K_{2}^{\prime}(x)-K_{2}(x)\right\|<\varepsilon_{2}, \\
\left\|\lambda_{i, 2}^{\prime}(x)-\lambda_{i, 2}(x)\right\|<\varepsilon_{2}, \\
\operatorname{dist}\left(\lambda_{i, 2}^{\prime}(x), \mathscr{A}\right)<\varepsilon_{2}, \\
\left\|\lambda_{i, 2}^{\prime}(x)-\lambda_{i, 1}(x)\right\|<C \sqrt[4]{2\left(\varepsilon_{1}+\varepsilon_{2}\right)+\varepsilon_{2}}<2 C \sqrt[4]{\varepsilon_{1}+\varepsilon_{2}} .
\end{gathered}
$$

Тогда поскольку

$$
\begin{array}{r}
\left\|K_{m-1}^{\prime}(x)-K_{m}(x)\right\| \leqslant\left\|K_{m-1}^{\prime}(x)-K_{m-1}(x)\right\|+\left\|K_{m-1}(x)-K(x)\right\| \\
+\left\|K(x)-K_{m}(x)\right\|<2 \varepsilon_{m-1}+\varepsilon_{m}
\end{array}
$$

то по индукции мы можем найти последовательность $K_{m}^{\prime}(x) \sim \operatorname{diag}\left(\left\{\lambda_{i, m}^{\prime}(x)\right\}\right)$ такую, что

$$
\begin{gathered}
\left\|K_{m}^{\prime}(x)-K_{m}(x)\right\|<\varepsilon_{m}, \\
\left\|\lambda_{i, m}^{\prime}(x)-\lambda_{i, m}(x)\right\|<\varepsilon_{m}, \\
\operatorname{dist}\left(\lambda_{i, m}^{\prime}(x), \mathscr{A}\right)<\varepsilon_{m} \\
\left\|\lambda_{i, m}^{\prime}(x)-\lambda_{i, m-1}(x)\right\|<C \sqrt[4]{2\left(2 \varepsilon_{m-1}+\varepsilon_{m}\right)+\varepsilon_{m}}<2 C \sqrt[4]{\varepsilon_{m-1}+\varepsilon_{m}} .
\end{gathered}
$$

Поскольку по (3.9)

$$
\left\|K_{m}^{\prime}(x)-K(x)\right\| \leqslant\left\|K_{m}^{\prime}(x)-K_{m}(x)\right\|+\left\|K_{m}(x)-K(x)\right\|<2 \varepsilon_{m}
$$


то последовательность операторов $K_{m}^{\prime}(x)$ сходится (по норме) к оператору $K(x)$, а из (3.11) видно, что для любого $i$ последовательности $\lambda_{i, m}^{\prime}(x)$ являются последовательностями Коши, если числа $\varepsilon_{m}$ стремятся к нулю достаточно быстро, именно, если сходится ряд $\sum_{m} \sqrt[4]{\varepsilon_{m-1}+\varepsilon_{m}}$. Поэтому сушествуют пределы

$$
\bar{\lambda}_{i}(x)=\lim _{m \rightarrow \infty} \lambda_{i, m}^{\prime}(x),
$$

и так как согласно $(3.10) \operatorname{dist}\left(\lambda_{i, m}^{\prime}(x), \mathscr{A}\right)$ стремится к нулю, то $\bar{\lambda}_{i}(x) \in \mathscr{A}$. Покажем, что $\bar{\lambda}_{i}(x)$ являются “собственными значениями" оператора $K(x)$. По теореме 1.3 из оценки $\left\|K_{m}^{\prime}(x)-K(x)\right\|<2 \varepsilon_{m}$ следует, что сушествуют унитарные операторы $U_{m} \in M_{n_{m}} \otimes \mathbf{A}$, отображающие "собственные векторы" $x_{i} \in H_{\mathbf{A}}^{*}$ оператора $K(x)$ в "собственные векторы" операторов $K_{m}^{\prime}(x)$ такие, что $\left\|U_{m}^{*} K_{m}^{\prime} U_{m}-K\right\|<2 \varepsilon_{m}$. Положим $\widetilde{K}_{m}=U_{m}^{*} K_{m}^{\prime} U_{m} \in M_{n_{m}} \otimes \mathbf{A}, \widetilde{K}_{m} \rightarrow K$. Тогда

$$
\widetilde{K}_{m} x_{i}=x_{i} \lambda_{i, m}^{\prime}
$$

Переходя к пределу в (3.12), получаем $K x_{i}=x_{i} \bar{\lambda}_{i}$. Теорема 3.1 доказана.

\section{§4. Пример: непрерывное поле алгебр врашения}

В заключение мы приводим пример $C^{*}$-алгебры без свойства слабой диагонализации. Пусть $X=[a, b]$ есть отрезок числовой прямой, содержащий целочисленную точку, например, 1 . Пусть $S$ - окружность, $C(X \times S)$ - $C^{*}$-алгебра непрерывных функций на цилиндре. Через $\alpha$ обозначим действие группы $\mathbb{Z}$ целых чисел на этой алгебре, определенное формулой

$$
(\alpha(n) f)(x, t)=f(x, t+n x),
$$

где $f(x, t) \in C(X \times S), x \in X, t \in S, n \in \mathbb{Z}$. Через $A_{X}$ обозначим скрешенное произведение

$$
C(X \times S) \rtimes_{\alpha} \mathbb{Z} .
$$

Как показано в [22], [23], алгебра $A_{X}$ является непрерывным полем алгебр врашения $A_{x}=A(x)$ над отрезком. Заметим, что непрерывное поле $\left(A(x), X, A_{X}\right)$ является непрерывным полем $C^{*}$-алгебр со следом, и для иррациональных $x$ $R R(A(x))=0$ и $\operatorname{tsr}(A(x))=1$ [19], [24]. Более того, для каждого $x \in X$ алгебра $A(x)$ обладает свойством слабой диагонализации. Унитарная группа алгебры $A(x)$ несвязна, но препятствие к диагонализации, по-видимому, связано не с этим. Заметим, что $A_{1} \cong C\left(\mathbf{T}^{2}\right)$ - коммутативная $C^{*}$-алгебра непрерывных функций на торе, поэтому она, в отличие от иррационального случая, не является алгеброй нулевого вешественного ранга, и гомоморфизм $K_{0}(A(1)) \rightarrow K_{0}(B(1))$ не инъективен. В $M_{2} \otimes A_{1}$ существует проектор, являющийся образующей Ботта в $K^{0}\left(\mathbf{T}^{2}\right)$. Этот проектор можно продолжить [24], [25] до непрерывного поля проекторов $p(x)$ в некоторой окрестности точки $1 \in X$ так, что для стандартного следа $\tau_{x}$ на $A_{X}$

$$
\tau_{x}(p(x))=1+x .
$$

Мы можем диагонализировать это поле проекторов в прямом интеграле факторов типа $\mathrm{II}_{1}, p(x) \sim \operatorname{diag}\left(\left\{\lambda_{1}(x), \lambda_{2}(x)\right\}\right)$, где $\lambda_{1}(x) \geqslant \lambda_{2}(x)$. Тогда для $x=1$ должно 
быть $\lambda_{1}(1)=1, \lambda_{2}(1)=0$. Для произвольных $x$ "собственные значения" $\lambda_{i}(x)$, $i=1,2$, должны быть проекторами. Если эти "собственные значения" являются непрерывными полями в $A_{X}$, то для всех $x$ должно быть $\lambda_{1}(x)=\lambda_{1}(1)=1$, $\lambda_{2}(x)=\lambda_{2}(1)=0$, следовательно,

$$
\tau_{x}\left(\operatorname{diag}\left(\left\{\lambda_{1}(x), \lambda_{2}(x)\right\}\right)\right)=1 .
$$

Но так как след инвариантен относительно унитарной эквивалентности, это противоречит (4.2), следовательно, $C^{*}$-алгебра $A_{X}$ не обладает свойством слабой диагонализации.

Отметим, что поскольку алгебры $A(x)$ удовлетворяют всем условиям теоремы 3.1 для плотного множества иррациональных точек в $X$, то, слегка изменяя наше доказательство, для любого заданного непрерывного поля операторов $K(x)$ и для любого $\varepsilon>0$ мы можем найти подмножество $X_{\varepsilon} \subset X$ такое, что мера множества $X \backslash X_{\varepsilon}$ не превосходит $\varepsilon$, и $K(x)$ является диагонализируемым на $X_{\varepsilon}$ с "непрерьвньми" "собственными значениями" (т.е. лежащими в $A_{X_{\varepsilon}}$ ).

\section{Список литературы}

1. Dixmier J. Les $C^{*}$-algèbres et leurs représentations. Paris: Gauthier-Villars, 1964.

2. Exel R., Loring T. A. Invariants of almost commuting unitaries // J. Funct. Anal. 1991. V. 95. P. 364-376.

3. Paschke W. L. Inner product modules over $B^{*}$-algebras // Trans. Amer. Math. Soc. 1973. V. 182. P. 443-468.

4. Paschke W. L. The double $B$-dual of an inner product module over a $C^{*}$-algebra // Canad. J. Math. 1974. V. 26. P. 1272-1280.

5. Rieffel M.A. Induced representations of $C^{*}$-algebras // Adv. Math. 1974. V. 13. P. 176-257.

6. Kasparov G. G. Hilbert $C^{*}$-modules: Theorems of Stinespring and Voiculescu // J. Operator Theory. 1980. V. 4. P. 133-150.

7. Мищенко A.C., Фоменко A. T. Индекс эллиптических операторов над $C^{*}$-алгебрами // Изв. АН СССР. Сер. матем. 1979. Т. 43. С. 831-859.

8. Lin H. Injective Hilbert $C^{*}$-modules // Pacific J. Math. 1992. V. 154. P. 131-164.

9. Lance E. C. Hilbert $C^{*}$-modules - a toolkit for operator algebraists // Lecture Notes. Leeds: University of Leeds, 1993.

10. Kadison R. V. Diagonalizing matrices // Amer. J. Math. 1984. V. 106. P. 1451-1468.

11. Grove K., Pedersen G.K. Diagonalizing matrices over $C(X) / /$ J. Funct. Anal. 1984. V. 59. P. $64-89$.

12. Murphy Q. J. Diagonality in $C^{*}$-algebras // Math. Z. 1990. V. 199. P. 279-284.

13. Zhang $S$. Diagonalizing projections in multiplier algebras and in matrices over a $C^{*}$-algebra // Pacific J. Math. 1990. V. 145. P. 181-200.

14. Frank M., Manuilov V. M. Diagonalizing "compact" operators on Hilbert $W^{*}$-modules // Z. Anal. Anwendungen. 1995. V. 14. P. 33-41.

15. Мануйлов В. М. Диагонализация компактных операторов в гильбертовых модулях над $W^{*}$-алгебрами конечного типа // УМН. 1994. Т. 49. № 2. С. 159-160.

16. Manuilov V.M. Diagonalization of compact operators in Hilbert modules over finite $W^{*}$-algebras // Ann. Global Anal. Geom. 1995. V. 13. P. 207-226.

17. Мануйлов B. М. Диагонализация компактных операторов в гильбертовых модулях над $C^{*}$-алгебрами нулевого вещественного ранга // Матем. заметки (в печати).

18. Brown L. G., Pedersen G. K. $C^{*}$-algebras of real rank zero // J. Funct. Anal. 1991. V. 99. P. 131-149.

19. Choi M.-D., Elliot G. A. Density of self-adjoint elements with finite spectrum in an irrational rotation $C^{*}$-algebra // Math. Scand. 1990. V. 67. P. 73-86. 
20. Bunce J., Deddens $J$. A family of simple $C^{*}$-algebras related to weighted shift operators // J. Funct. Anal. 1975. V. 19. P. 13-24.

21. Sunder V.S., Thomsen $K$. Unitary orbits of selfadjoints in some $C^{*}$-algebras // Houston J. Math. 1992. V. 18. P. 127-137.

22. Rieffel $M$. A. Continuous fields of $C^{*}$-algebras coming from group cocycles and actions // Math. Ann. 1989. V. 283. P. 631-643.

23. Elliott G.A., Natsume T., Nest R. The Heisenberg group and $K$-theory // Københavns Universitet Preprint Series. № 25, 1992.

24. Rieffel M. A. $C^{*}$-algebras associated with irrational rotations // Pacific J. Math. 1981. V. 93. P. 415-429.

25. Мануйлов B. M. О группе $K_{0}$ непрерьвного семейства алгебр $A_{\theta} / /$ УМН. 1989. Т. 44. №3. C. $163-164$.

Московский государственньй университет им. М.В. Ломоносова
Поступила в редакцию 10.12 .1996 